\title{
Observation of muon intensity variations by season with the MINOS far detector
}

P. Adamson, ${ }^{8}$ C. Andreopoulos, ${ }^{19}$ K. E. Arms, ${ }^{14}$ R. Armstrong, ${ }^{12}$ D. J. Auty, ${ }^{23}$ D. S. Ayres, ${ }^{1}$ C. Backhouse, ${ }^{17}$ J. Barnett, ${ }^{17}$ G. Barr, ${ }^{17}$ W. L. Barrett, ${ }^{29}$ B. R. Becker, ${ }^{14}$ M. Bishai, ${ }^{4}$ A. Blake, ${ }^{6}$ B. Bock, ${ }^{15}$ G. J. Bock, ${ }^{8}$ D. J. Boehnlein, ${ }^{8}$ D. Bogert, ${ }^{8}$ C. Bower, ${ }^{12}$ S. Cavanaugh, ${ }^{9}$ J. D. Chapman, ${ }^{6}$ D. Cherdack, ${ }^{26}$ S. Childress, ${ }^{8}$ B. C. Choudhary, ${ }^{8 *}$ J. H. Cobb, ${ }^{17}$ S. J. Coleman, ${ }^{28}$ D. Cronin-Hennessy, ${ }^{14}$ A. J. Culling, ${ }^{6}$ I. Z. Danko, ${ }^{18}$ J. K. de Jong, ${ }^{17,11}$ N. E. Devenish, ${ }^{23}$ M. V. Diwan, ${ }^{4}$ M. Dorman, ${ }^{13}$ C. O. Escobar, ${ }^{7}$ J. J. Evans, ${ }^{13,17}$ E. Falk, ${ }^{23}$ G. J. Feldman, ${ }^{9}$ T. H. Fields, ${ }^{1}$ M. V. Frohne, ${ }^{10,3}$ H. R. Gallagher, ${ }^{26}$ A. Godley, ${ }^{21}$ M. C. Goodman, ${ }^{1}$ P. Gouffon, ${ }^{20}$ R. Gran, ${ }^{15}$ E. W. Grashorn,,${ }^{14,15,}{ }^{\dagger}$ K. Grzelak,${ }^{27,17}$ A. Habig, ${ }^{15}$ D. Harris,${ }^{8}$ P. G. Harris, ${ }^{23}$ J. Hartnell, ${ }^{23,19}$ R. Hatcher, ${ }^{8}$ K. Heller, ${ }^{14}$ A. Himmel, ${ }^{5}$ A. Holin, ${ }^{13}$ J. Hylen, ${ }^{8}$ G. M. Irwin, ${ }^{22}$ Z. Isvan, ${ }^{18}$ D. E. Jaffe, ${ }^{4}$ C. James, ${ }^{8}$ D. Jensen, ${ }^{8}$ T. Kafka, ${ }^{26}$ S. M. S. Kasahara, ${ }^{14}$ G. Koizumi, ${ }^{8}$ S. Kopp,${ }^{25}$ M. Kordosky, ${ }^{28,13}$ K. Korman, ${ }^{15}$ D. J. Koskinen, ${ }^{13,15, *}$ Z. Krahn, ${ }^{14}$ A. Kreymer, ${ }^{8}$ K. Lang, ${ }^{25}$ J. Ling, ${ }^{21}$ P. J. Litchfield,${ }^{14}$ L. Loiacono, ${ }^{25}$ P. Lucas, ${ }^{8}$ J. Ma, ${ }^{25}$ W. A. Mann, ${ }^{26}$ M. L. Marshak, ${ }^{14}$ J. S. Marshall, ${ }^{6}$ N. Mayer, ${ }^{12}$ A. M. McGowan,,${ }^{14,8}$ R. Mehdiyev, ${ }^{25}$ J. R. Meier, ${ }^{14}$ M. D. Messier, ${ }^{12}$ C. J. Metelko, ${ }^{19}$ D. G. Michael,,${ }^{5, \mid}$ W. H. Miller, ${ }^{14}$ S. R. Mishra, ${ }^{21}$ J. Mitchell, ${ }^{6}$ C. D. Moore, ${ }^{8}$ J. Morfín,${ }^{8}$ L. Mualem, ${ }^{5}$ S. Mufson, ${ }^{12}$ J. Musser, ${ }^{12}$ D. Naples, ${ }^{18}$ J. K. Nelson, ${ }^{28}$ H. B. Newman, ${ }^{5}$ R. J. Nichol, ${ }^{13}$ T. C. Nicholls, ${ }^{19}$ J. P. Ochoa-Ricoux,${ }^{5}$ W. P. Oliver, ${ }^{26}$ T. Osiecki, ${ }^{25}$ R. Ospanov, ${ }^{25,}$, S. Osprey, ${ }^{17}$ J. Paley, ${ }^{12}$ R. B. Patterson, ${ }^{5}$ T. Patzak, ${ }^{30}$ G. Pawloski, ${ }^{22}$ G. F. Pearce, ${ }^{19}$ E. A. Peterson, ${ }^{14}$ R. Pittam, ${ }^{17}$ R. K. Plunkett, ${ }^{8}$ A. Rahaman, ${ }^{21}$ R. A. Rameika, ${ }^{8}$ T. M. Raufer, ${ }^{19,17}$ B. Rebel, ${ }^{8}$ J. Reichenbacher, ${ }^{1}$ P. A. Rodrigues,${ }^{17}$ C. Rosenfeld, ${ }^{21}$ H. A. Rubin, ${ }^{11}$ V. A. Ryabov ${ }^{31}$ M. C. Sanchez, ${ }^{1,9}$ N. Saoulidou, ${ }^{8}$ J. Schneps, ${ }^{26}$ P. Schreiner, ${ }^{3}$ P. Shanahan,${ }^{8}$ W. Smart ${ }^{8}$ C. Smith, ${ }^{23,5}$ A. Sousa, ${ }^{9,17}$ B. Speakman, ${ }^{14}$ P. Stamoulis, ${ }^{2}$ M. Strait, ${ }^{14}$ N. Tagg,,${ }^{16,26}$ R. L. Talaga, ${ }^{1}$ J. Thomas, ${ }^{13}$ M. A. Thomson, ${ }^{6}$ J. L. Thron,,${ }^{1, * *}$ G. Tinti, ${ }^{17}$ R. Toner, ${ }^{6}$ V. A. Tsarev,${ }^{31}$ G. Tzanakos, ${ }^{2}$ J. Urheim, ${ }^{12}$ P. Vahle,${ }^{28,13}$ B. Viren, ${ }^{4}$ M. Watabe,${ }^{24}$ A. Weber, ${ }^{17}$ R. C. Webb,${ }^{24}$ N. West, ${ }^{17}$ C. White, ${ }^{11}$ L. Whitehead, ${ }^{4}$ S. G. Wojcicki, ${ }^{22}$ D. M. Wright, ${ }^{32}$ T. Yang, ${ }^{22}$ M. Zois, ${ }^{2}$ K. Zhang, ${ }^{4}$ and R. Zwaska ${ }^{8}$

\section{(MINOS Collaboration)}

\author{
${ }^{1}$ Argonne National Laboratory, Argonne, Illinois 60439, USA \\ ${ }^{2}$ Department of Physics, University of Athens, GR-15771 Athens, Greece \\ ${ }^{3}$ Physics Department, Benedictine University, Lisle, Illinois 60532, USA \\ ${ }^{4}$ Brookhaven National Laboratory, Upton, New York 11973, USA \\ ${ }^{5}$ Lauritsen Laboratory, California Institute of Technology, Pasadena, California 91125, USA \\ ${ }^{6}$ Cavendish Laboratory, University of Cambridge, Madingley Road, Cambridge CB3 OHE, United Kingdom \\ ${ }^{7}$ Universidade Estadual de Campinas, IFGW-UNICAMP, CP 6165, 13083-970, Campinas, SP, Brazil \\ ${ }^{8}$ Fermi National Accelerator Laboratory, Batavia, Illinois 60510, USA \\ ${ }^{9}$ Department of Physics, Harvard University, Cambridge, Massachusetts 02138, USA \\ ${ }^{10}$ Holy Cross College, Notre Dame, Indiana 46556, USA \\ ${ }^{11}$ Physics Division, Illinois Institute of Technology, Chicago, Illinois 60616, USA \\ ${ }^{12}$ Indiana University, Bloomington, Indiana 47405, USA \\ ${ }^{13}$ Department of Physics and Astronomy, University College London, Gower Street, London WC1E 6BT, United Kingdom \\ ${ }^{14}$ University of Minnesota, Minneapolis, Minnesota 55455, USA \\ ${ }^{15}$ Department of Physics, University of Minnesota- Duluth, Duluth, Minnesota 55812, USA \\ ${ }^{16}$ Otterbein College, Westerville, Ohio 43081, USA \\ ${ }^{17}$ Department of Physics, University of Oxford, Oxford OX1 3RH, United Kingdom \\ ${ }^{18}$ Department of Physics and Astronomy, University of Pittsburgh, Pittsburgh, Pennsylvania 15260, USA \\ ${ }^{19}$ Rutherford Appleton Laboratory, Science and Technology Facilities Council, Harwell Science and Innovation Campus, \\ Didcot, OX11 OQX, United Kingdom \\ ${ }^{20}$ Instituto de Física, Universidade de São Paulo, CP 66318, 05315-970, São Paulo, SP, Brazil \\ ${ }^{21}$ Department of Physics and Astronomy, University of South Carolina, Columbia, South Carolina 29208, USA \\ ${ }^{22}$ Department of Physics, Stanford University, Stanford, California 94305, USA \\ ${ }^{23}$ Department of Physics and Astronomy, University of Sussex, Falmer, Brighton BN1 9QH, United Kingdom \\ ${ }^{24}$ Physics Department, Texas A\&M University, College Station, Texas 77843, USA \\ ${ }^{25}$ Department of Physics, University of Texas at Austin, 1 University Station C1600, Austin, Texas 78712, USA \\ ${ }^{26}$ Physics Department, Tufts University, Medford, Massachusetts 02155, USA \\ ${ }^{27}$ Department of Physics, University of Warsaw, Hoża 69, PL-00-681 Warsaw, Poland \\ ${ }^{28}$ Department of Physics, College of William \& Mary, Williamsburg, Virginia 23187, USA \\ ${ }^{29}$ Physics Department, Western Washington University, Bellingham, Washington 98225, USA \\ ${ }^{30}$ APC-Université Paris 7 Denis Diderot, 10, rue Alice Domon et Léonie Duquet, F-75205 Paris Cedex 13, France
}


${ }^{31}$ Nuclear Physics Department, Lebedev Physical Institute, Leninsky Prospect 53, 119991 Moscow, Russia

${ }^{32}$ Lawrence Livermore National Laboratory, Livermore, California 94550, USA

(Received 12 September 2009; revised manuscript received 19 November 2009; published 6 January 2010)

\begin{abstract}
The temperature of the upper atmosphere affects the height of primary cosmic ray interactions and the production of high-energy cosmic ray muons which can be detected deep underground. The MINOS far detector at Soudan, MN, has collected over $67 \times 10^{6}$ cosmic ray induced muons. The underground muon rate measured over a period of five years exhibits a $4 \%$ peak-to-peak seasonal variation which is highly correlated with the temperature in the upper atmosphere. The coefficient, $\alpha_{T}$, relating changes in the muon rate to changes in atmospheric temperature was found to be $\alpha_{T}=0.873 \pm 0.009$ (stat) \pm 0.010 (syst). Pions and kaons in the primary hadronic interactions of cosmic rays in the atmosphere contribute differently to $\alpha_{T}$ due to the different masses and lifetimes. This allows the measured value of $\alpha_{T}$ to be interpreted as a measurement of the $K / \pi$ ratio for $E_{p} \gtrsim 7 \mathrm{TeV}$ of $0.12_{-0.05}^{+0.07}$, consistent with the expectation from collider experiments.
\end{abstract}

DOI: 10.1103/PhysRevD.81.012001

PACS numbers: 95.55.Vj, 98.70.Sa, 98.70.Vc

\section{INTRODUCTION}

When very high energy cosmic rays interact in the stratosphere, mesons are produced in the primary hadronic interaction. These mesons either interact and produce lower energy hadronic cascades, or decay into high-energy muons which can be observed deep underground. While the temperature of the troposphere varies considerably within the day, the temperature of the stratosphere remains nearly constant, usually changing on the time scale of seasons (with the exception of the occasional sudden stratospheric warming [1]). An increase in temperature of the stratosphere causes a decrease in density. This reduces the chance of meson interaction, resulting in a larger fraction decaying to produce muons. This results in a higher muon rate observed deep underground [2-4]. The majority of muons detected in the MINOS far detector are produced in the decay of pions, although the decays of kaons must be considered for a more complete description of the flux [5].

MINOS is a long baseline neutrino oscillation experiment [6,7], with a neutrino source and near detector at Fermi National Accelerator Laboratory in Batavia, IL, and a far detector at the Soudan Underground Mine State Park in northern Minnesota. This paper describes cosmic ray data taken in the far detector, a scintillator and steel tracking calorimeter located $0.72 \mathrm{~km}$ underground (2100 mwe, meters water equivalent) [8]. It has a 5.4 kton mass and a

\footnotetext{
*Now at Department of Physics and Astrophysics, University of Delhi, Delhi 110007, India.

${ }^{\dagger}$ Now at Center for Cosmology and Astro Particle Physics, Ohio State University, Columbus, $\mathrm{OH} 43210$, USA.

${ }^{*}$ Now at Department of Physics, Penn State University, State College, PA 16802, USA.

${ }^{\S}$ Now at Physics Department, St. John Fisher College, Rochester, NY 14618, USA.

"Deceased.

IINow at Department of Physics and Astronomy, University of Pennsylvania, Philadelphia, PA 19104-6396, USA.

**Now at Nuclear Nonproliferation Division, Threat Reduction Directorate, Los Alamos National Laboratory, Los Alamos, NM 87545, USA.
}

$6.91 \times 10^{6} \mathrm{~cm}^{2} \mathrm{sr}$ [9] acceptance. Because of its depth, MINOS detects cosmic ray muons with energy at the surface, $E_{\mu}>0.73 \mathrm{TeV}$. These high-energy muons are mostly the result of the decays of the mesons produced in the primary hadronic interaction. This, coupled with the large acceptance, makes it possible to detect small seasonal temperature fluctuations in the upper atmosphere. The far detector is the deepest underground detector with a magnetic field, allowing the separation of particles by charge.

The MINOS data are correlated with atmospheric temperature measurements at the Soudan site provided by the European Centre for Medium-Range Weather Forecasts (ECMWF) [10]. This temperature data set has higher precision than any other used for the seasonal variation analysis [2,4,11-17]. The $67.32 \times 10^{6}$ muon events used in this analysis were collected over five years, from August 1, 2003 to July 31, 2008, a period that includes five complete annual cycles. The seasonal variations in muon intensity were compared to a theoretical model which extends the pion-only model of [3] to include the contribution from kaons.

\section{EXPERIMENTAL EFFECTIVE TEMPERATURE COEFFICIENT}

\section{A. Experimental intensity}

The underground muon intensity depends on the threshold energy $E_{\text {th }}$ and the cosine of the zenith angle $\theta$. The change in underground muon intensity variations as a function of temperature was derived following the formalism of $[2,3]$. The change in the surface muon intensity, $\Delta I_{\mu}(E, \cos \theta)$ occurring at the MINOS far detector site can be written as

$$
\Delta I_{\mu}=\int_{0}^{\infty} d X W(X) \Delta T(X)
$$

where $\Delta T(X)$ is the change in atmospheric temperature at atmospheric depth $X$, and the weight $W(X)$ reflects the temperature dependence of the production of mesons in 
the atmosphere and their decay into muons that can be observed in the far detector. A temperature coefficient $\alpha(X)$ can be defined as

$$
\alpha(X)=\frac{T(X)}{I_{\mu}^{0}} W(X),
$$

where $I_{\mu}^{0}$ is the muon intensity evaluated at a given value of atmospheric temperature $T_{0}$. The phenomenological relationship between the atmospheric temperature fluctuations and muon intensity variations can now be written as

$$
\frac{\Delta I_{\mu}}{I_{\mu}^{0}}=\int_{0}^{\infty} d X \alpha(X) \frac{\Delta T(X)}{T_{0}} .
$$

The atmosphere consists of many levels that vary continuously in both temperature and pressure. To simplify the calculations, the atmosphere is approximated by an isothermal body with an effective temperature, $T_{\text {eff }}$, obtained from a weighted average over the atmospheric depth:

$$
T_{\text {eff }}=\frac{\int_{0}^{\infty} d X T(X) W(X)}{\int_{0}^{\infty} d X W(X)}
$$

An "effective temperature coefficient," $\alpha_{T}$ can then be defined

$$
\alpha_{T}=\frac{T_{\mathrm{eff}}}{I_{\mu}^{0}} \int_{0}^{\infty} d X W(X) .
$$

With these definitions in place, the relationship between atmospheric temperature fluctuations and muon intensity variations can now be written as

$$
\frac{\Delta I_{\mu}}{I_{\mu}^{0}}=\alpha_{T} \frac{\Delta T_{\mathrm{eff}}}{T_{\mathrm{eff}}} .
$$

The configuration and geometric acceptance of the far detector remain constant over time. Therefore, the rate, $R_{\mu}$ of muons observed in the detector is proportional to the incident muon intensity and varies with the effective atmospheric temperature as follows:

$$
\frac{\Delta R_{\mu}}{\left\langle R_{\mu}\right\rangle}=\alpha_{T} \frac{\Delta T_{\text {eff }}}{\left\langle T_{\text {eff }}\right\rangle} .
$$

In practice, the observed muon rates and the temperature data are averaged over the period of a day. The effective temperature is obtained from a weighted average of temperature measurements obtained at a set of discrete pressure levels.

The weight $W(X)$ can be written as the sum $W^{\pi}+W^{K}$, representing the contribution of pions and kaons to the overall variation in muon intensity. The weights $W^{\pi, K}$ are given by $[18,19]$

$$
W^{\pi, K}(X) \simeq \frac{\left(1-X / \Lambda_{\pi, K}^{\prime}\right)^{2} e^{-X / \Lambda_{\pi, K}} A_{\pi, K}^{1}}{\gamma+(\gamma+1) B_{\pi, K}^{1} K(X)\left(\left\langle E_{\mathrm{th}} \cos \theta\right\rangle / \epsilon_{\pi, K}\right)^{2}},
$$

where

$$
K(X) \equiv \frac{\left(1-X / \Lambda_{\pi, K}^{\prime}\right)^{2}}{\left(1-e^{-X / \Lambda_{\pi, K}^{\prime}}\right) \Lambda_{\pi, K}^{\prime} / X}
$$

The parameters $A_{\pi, K}^{1}$ include the amount of inclusive meson production in the forward fragmentation region, masses of mesons and muons, and muon spectral index; the input values are $A_{\pi}^{1}=1$ and $A_{K}^{1}=0.38 \cdot r_{K / \pi}[18,19]$, where $r_{K / \pi}$ is the $K / \pi$ ratio. The parameters $B_{\pi, K}^{1}$ reflect the relative atmospheric attenuation of mesons; the threshold energy, $E_{\mathrm{th}}$, is the energy required for a muon to survive to a particular depth; the attenuation lengths for the cosmic ray primaries, pions and kaons are $\Lambda_{N}, \Lambda_{\pi}$ and $\Lambda_{K}$ respectively with $1 / \Lambda_{\pi, K}^{\prime} \equiv 1 / \Lambda_{N}-1 / \Lambda_{\pi, K}$. The muon spectral index is given by $\gamma$. The meson critical energy, $\epsilon_{\pi, K}$, is the meson energy for which decay and interaction have an equal probability. Since the distribution has a long tail (Fig. 3), the value of $\left\langle E_{\mathrm{th}} \cos \theta\right\rangle$ used here is the median. The values for these parameters can be found in Table I.

Since the temperature is measured at discrete levels, the integral is represented by a sum over the atmospheric levels $X_{n}$ :

$$
T_{\mathrm{eff}} \simeq \frac{\sum_{n=0}^{N} \Delta X_{n} T\left(X_{n}\right)\left(W_{n}^{\pi}+W_{n}^{K}\right)}{\sum_{n=0}^{N} \Delta X_{n}\left(W_{n}^{\pi}+W_{n}^{K}\right)}
$$

where $W_{n}^{\pi, K}$ is $W^{\pi, K}$ evaluated at $X_{n}$. The temperature and pressure vary continuously through the atmosphere. Figure 1 (solid line) shows the average temperature from 2003-2008 above Soudan as a function of pressure level in the atmosphere [10]. The height axis on the right represents the average log-pressure height, the height of a pressure level relative to the surface pressure, corresponding to the average temperatures plotted here. The dashed line is the weight as a function of pressure level $W(X)$, obtained from Eq. (8) and normalized to one, used to calculate the effective temperature. The dashed weight curve in Fig. 1 shows that the temperature fluctuations higher in the atmosphere

TABLE I. Input $W(X)$ parameter values.

\begin{tabular}{lc}
\hline \hline Parameter & Value \\
\hline$A_{\pi}^{1}$ & $1[18,19]$ \\
$A_{K}^{1}$ & $0.38 \cdot r_{K / \pi}[18,19]$ \\
$r_{K / \pi}$ & $0.149[20] \pm 0.06[21]$ \\
$B_{\pi}^{1}$ & $1.460 \pm 0.007[18,19]$ \\
$B_{K}^{1}$ & $1.740 \pm 0.028[18,19]$ \\
$\Lambda_{N}$ & $120 \mathrm{~g} / \mathrm{cm}^{2}[20]$ \\
$\Lambda_{\pi}$ & $180 \mathrm{~g} / \mathrm{cm}^{2}[20]$ \\
$\Lambda_{K}$ & $160 \mathrm{~g} / \mathrm{cm}^{2}[20]$ \\
$\left\langle E_{\text {th }} \cos \theta\right\rangle$ & $0.785 \pm 0.14 \mathrm{TeV}$ \\
$\gamma$ & $1.7 \pm 0.1[5]$ \\
$\epsilon_{\pi}$ & $0.114 \pm 0.003 \mathrm{TeV}[18,19]$ \\
$\epsilon_{K}$ & $0.851 \pm 0.014 \mathrm{TeV}[18,19]$ \\
\hline \hline
\end{tabular}




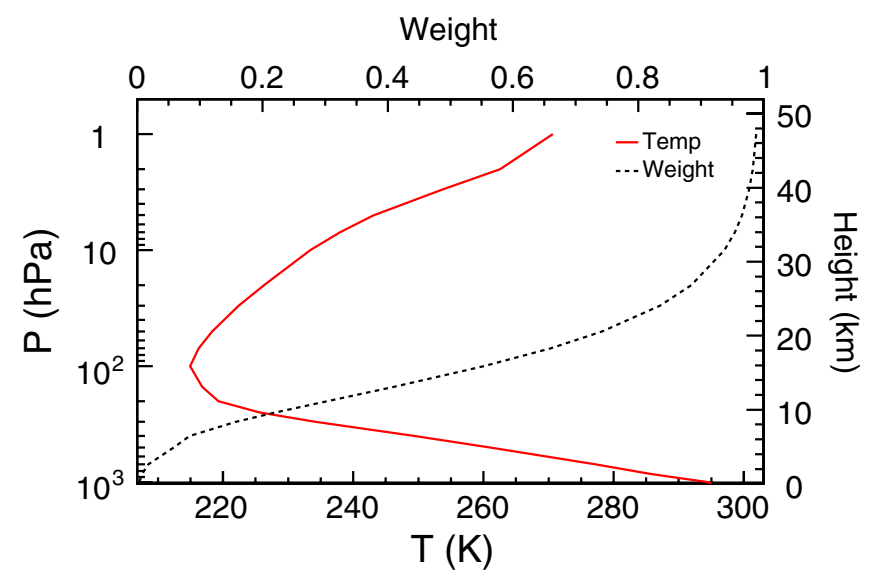

FIG. 1 (color online). The $5 \mathrm{yr}$ average temperature at various pressure levels (solid line). The range is from $1000 \mathrm{hPa}(1 \mathrm{hPa}=$ $1.019 \mathrm{~g} / \mathrm{cm}^{2}$ ), near the Earth's surface, to $1 \mathrm{hPa}$ (nearly $50 \mathrm{~km}$ ), near the top of the stratosphere. The height axis on the right represents the average log-pressure height corresponding to the average temperatures plotted here. The dashed line is the weight as a function of pressure level $(X)$ used to find $T_{\text {eff }}$. The weights are determined by Eq. (8), normalized to one.

have a greater effect on the production of muons visible at a depth of 2100 mwe. High-energy mesons produced at the top of the atmosphere are more likely to decay, producing muons visible to MINOS, than those produced lower in the atmosphere. Note that the expression used to calculate $T_{\text {eff }}$ in the pion scaling limit, ignoring the kaon contribution, is the same as the MACRO calculation [3]. The effective temperature coefficient, $\alpha_{T}$, is a function of both the muon threshold energy and the $K / \pi$ ratio. As the energy increases, the muon intensity becomes more dependent on the meson critical energy, which in turn is proportional to the atmospheric temperature. The effective temperature coefficient thus reflects the fraction of mesons that are sensitive to atmospheric temperature variations, and for energies much greater than the critical energy, the value of $\alpha_{T}$ approaches unity. At the depth of the MINOS far detector, the vertical muon threshold energy lies between the pion and kaon critical energies. Therefore, because the muon energy is close to the parent meson's energy, a larger $K / \pi$ ratio results in a smaller value of $\alpha_{T}$.

\section{B. Data}

The muon data for this analysis were accumulated over a 5 yr span, beginning on August 1, 2003. Data quality cuts were performed to ensure a clean sample of muons (preanalysis cuts) [5]

(1) Require that all detector readout and subsystems were functioning normally

(2) Remove runs with anomalous cosmic ray rates, greater than $1 \mathrm{~Hz}$

(3) Remove events that had many hits assigned to incorrect channels (properly demultiplexed [5])

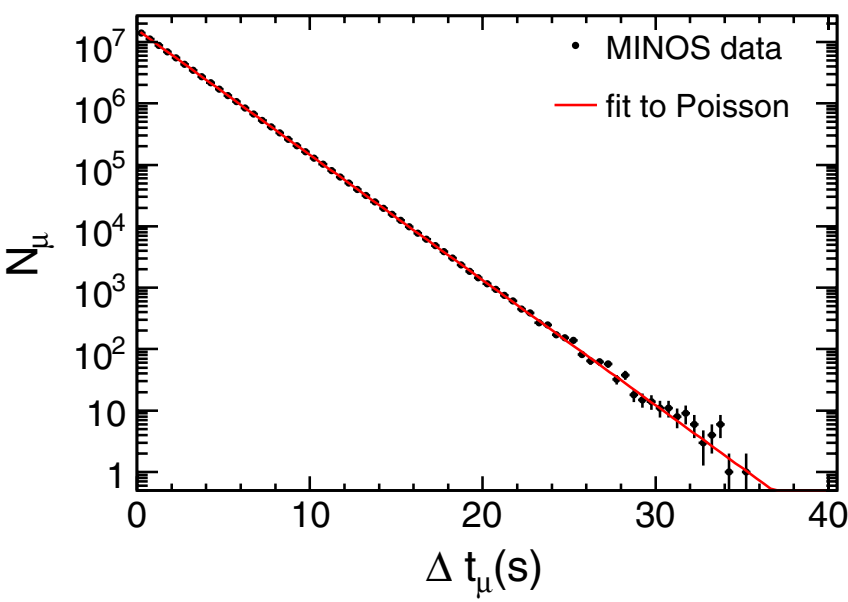

FIG. 2 (color online). The time between consecutive cosmic ray muon arrivals, fit with a Poisson distribution. The fit gives $\chi^{2} / N_{\text {d.o.f. }}=55.2 / 68 ; \quad\left\langle R_{\mu}\right\rangle=0.4692 \pm 0.0001 \mathrm{~Hz} \quad$ (from slope). The Poissonian nature of the muon arrival times demonstrates the absence of short time scale systematic effects on the data.

(4) Remove muons induced by NuMI beam interactions with timing cuts [6].

After all cuts were applied the initial sample of $68.66 \times$ $10^{6}$ muons was reduced to $67.32 \times 10^{6}$ muons [18]. A plot of the time between consecutive muon arrivals in the MINOS data is shown in Fig. 2. The distribution is well described by a Poisson distribution $[18,22]$ with mean rate $\left\langle R_{\mu}\right\rangle=0.4692 \pm 0.0001 \mathrm{~Hz}$, demonstrating the absence of short-time-scale systematic effects on the data. The average muon rate was calculated for each day by dividing the number of observed muons by the detector live time.

The energy spectra for the observed muons can be seen in Fig. 3. The solid line is $E_{\mathrm{th}} \cos \theta$, which was used to determine the value used in Eq. (8). The dashed line is the distribution of muon surface energies, $E_{\mu}$, in the far detector, which has a much longer tail than the distribution of threshold energies. Also shown are $E_{\mathrm{th}} \cos \theta$ (CS) (dotdashed line), the distribution of $E_{\mathrm{th}} \cos \theta$ after chargeseparation cuts have been applied, and $E_{\mu}(\mathrm{CS})$ (dotted line), the distribution of $E_{\mu}$ after the charge-separation cuts (see Sec. IIC) have been applied. Note that the charge-separation cuts have been applied, but the distributions shown include both muon species. The $E_{\mathrm{th}} \cos \theta$ distribution is peaked, with a median value $\left\langle E_{\mathrm{th}} \cos \theta\right\rangle=$ $0.795 \pm 0.14 \mathrm{TeV}$. This distribution, with its rapid falloff, reflects the rock overburden surrounding the far detector.

The temperature data for the Soudan site were obtained from ECMWF, which collates a number of different types of observations (e.g. surface, satellite and upper air sounding) at many locations around the globe, and uses a global atmospheric model to interpolate to a particular location. For this analysis, the ECMWF model produced atmos- 


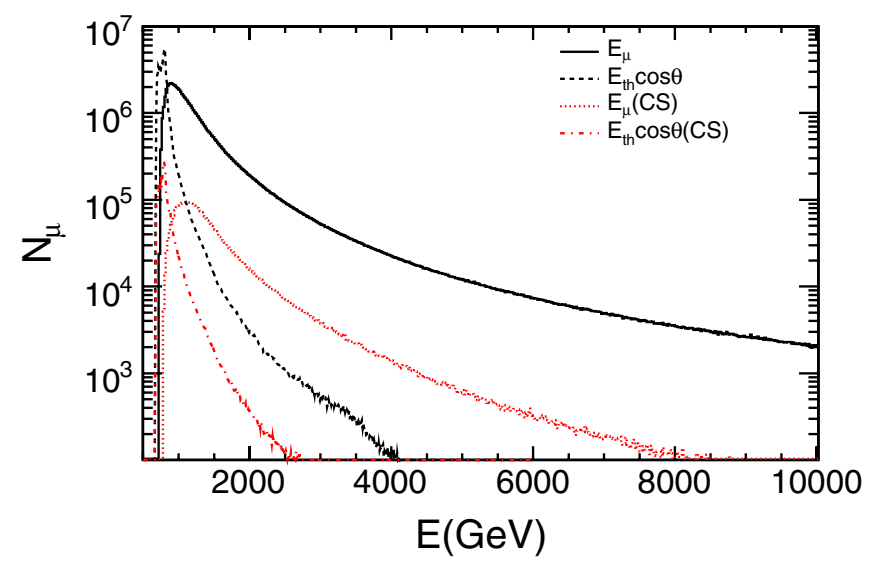

FIG. 3 (color online). A plot of the energy spectra observed in the far detector. The dashed line is $E_{\mathrm{th}} \cos \theta$, which was used to determine the value used in Eq. (8). The solid line is the distribution of muon surface energies, $E_{\mu}$ in far detector. Also shown are $E_{\text {th }} \cos \theta(\mathrm{CS})$ (dot-dashed line), the distribution of $E_{\mathrm{th}} \cos \theta$ after charge-separation cuts have been applied, and $E_{\mu}(\mathrm{CS})$ (dotted line), the distribution of $E_{\mu}$ after the chargeseparation cuts (see Sec. IIC) have been applied. Note that the charge-separation cuts have been applied, but the distributions shown include both muon species.

pheric temperatures at 21 discrete pressure levels: 1000, $925,850,700,500,400,300,250,200,150,100,70,50$, $30,20,10,7,5,3,2$ and $1 \mathrm{hPa}\left(1 \mathrm{hPa}=1.019 \mathrm{~g} / \mathrm{cm}^{2}\right)$, at four times, $0000 \mathrm{~h}, 0600 \mathrm{~h}, 1200 \mathrm{~h}$ and $1800 \mathrm{~h}$ each day. The effective temperature, $T_{\text {eff }}$, was calculated four times each day using Eq. (10). A mean value $\left\langle T_{\text {eff }}\right\rangle$ and error were obtained from these four daily measurements. The ECMWF temperature data were cross-checked using the Integrated Global Radiosonde Archive (IGRA) of temperature measurements [23]. The distribution of the differences between ECMWF and IGRA temperature values at International Falls, $\mathrm{MN}$, was well described by a Gaussian distribution with $\sigma=0.31 \mathrm{~K}$.

Figure 4 shows the percentage deviation in the mean daily muon rate, $\Delta R_{\mu}$, over the entire set of data, with statistical error bars. A typical day at $\left\langle R_{\mu}\right\rangle=0.4692 \mathrm{~Hz}$ yields $\sim 40000$ muons, resulting in error bars of order $0.5 \%$. The variation with season can be seen, with maxima in August and minima in February. These maxima peak at rates that are within $0.5 \%$ of each other. For the $5 \mathrm{yr}$ period $\left\langle T_{\text {eff }}\right\rangle=221.93 \mathrm{~K}$. The distribution of $\Delta T_{\text {eff }}$ over the data period can be seen in Fig. 5, with strong periodic seasonal correlation with the data. There is also striking correspondence between Figs. 4 and 5 for small term maxima and minima over a few days' span.

A plot of $\Delta R_{\mu} /\left\langle R_{\mu}\right\rangle\left(\Delta T_{\text {eff }}\right)$ was produced (Fig. 6) for each day's $\Delta R_{\mu}$ and $\Delta T_{\text {eff }}$ data to quantify the daily correlation between rate and temperature. To find the value for $\alpha_{T}$, a linear regression was performed using the MINUIT [24] fitting package. This package performs a linear re-

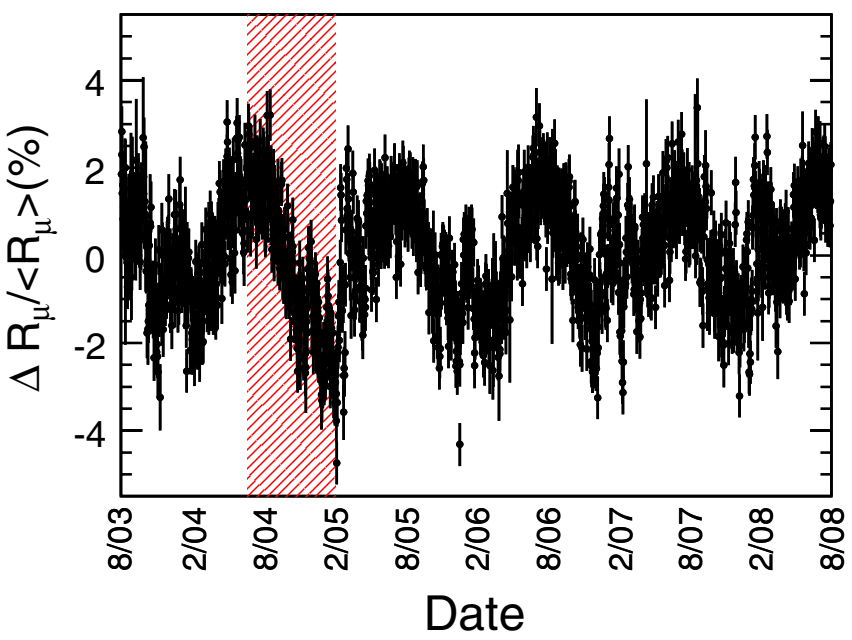

FIG. 4 (color online). The daily deviation from the mean rate of cosmic ray muon arrivals from $8 / 03-8 / 08$, shown here with statistical error bars. The periodic fluctuations have the expected maxima in August, minima in February. The hatched region indicates the period of time when the detector ran with the magnetic field reversed from the normal configuration.

gression accounting for error bars on both the $x$ and $y$ axis using a numerical minimization method. The result of this fit is a slope of $\alpha_{T}=0.873 \pm 0.009$ (statistical errors only), and the correlation coefficient ( $R$ value) between these two distributions is 0.90 .

The effects of systematic uncertainties were evaluated by modifying parameters and recalculating $\alpha_{T}$. Table II shows the difference in calculated $\alpha_{T}$ for the modified parameters. The largest systematic errors are (a) the \pm 0.06 uncertainty in meson production ratio [21]; (b) the $\pm 0.31 \mathrm{~K}$ uncertainty in mean effective temperature, esti-

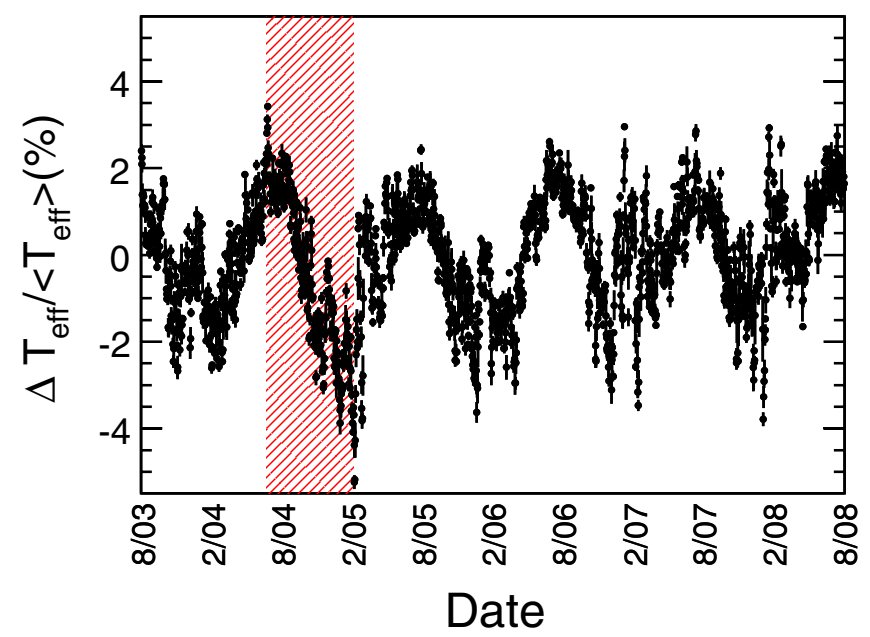

FIG. 5 (color online). The daily deviation from the mean effective temperature over a period of five years, beginning when the far detector was complete, 08/03-08/08. The hatched region indicates the period of time when the detector ran with the magnetic field reversed from the normal configuration. 


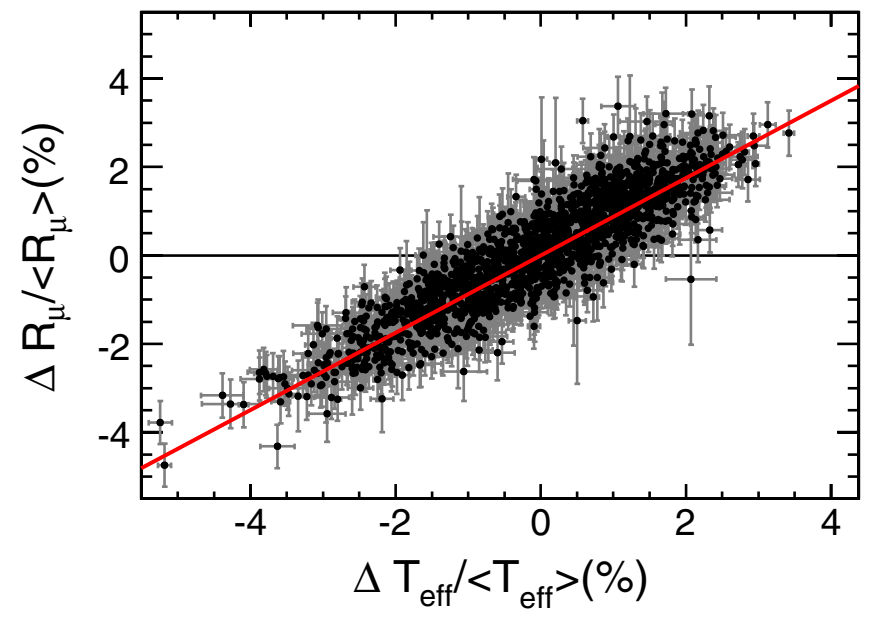

FIG. 6 (color online). A plot of $\Delta R_{\mu} /\left\langle R_{\mu}\right\rangle$ as a function of $\Delta T_{\text {eff }} /\left\langle T_{\text {eff }}\right\rangle$ for single muons, fit by a line with the $y$ intercept fixed at 0 . The fit has a $\chi^{2} / N_{\text {d.o.f. }}=1959 / 1797$, and the slope is $\alpha_{T}=0.873 \pm 0.009$.

mated by comparing ECMWF temperatures at International Falls, MN, to those of the IGRA [23] measurements; (c) the $\pm 0.14 \mathrm{TeV}$ uncertainty in muon threshold energy, estimated from uncertainties in the rock overburden above the far detector. To estimate this uncertainty, the rock map was adjusted up by $10 \%$ and $\left\langle E_{\mathrm{th}} \cos \theta\right\rangle$ was calculated, then the rock map was adjusted down by $10 \%$ and $\left\langle E_{\mathrm{th}} \cos \theta\right\rangle$ was again recalculated. The uncertainty was then calculated from the difference between $\left\langle E_{\mathrm{th}} \cos \theta\right\rangle$ and these adjusted values. These systematic errors were added in quadrature and are included with the error from the linear fit to obtain the experimental value of $\alpha_{T}=0.873 \pm 0.009$ (stat) \pm 0.010 (syst).

\section{Charge separated}

To obtain a sample of events with well-measured charge sign, further selection requirements were applied to the length and radius of curvature of muon tracks. These cuts, taken from previous investigations of the muon charge ratio at MINOS [5], have the effect of reducing the energy distribution at the Earth's surface of the selected muon sample.

TABLE II. Systematic errors on the experimental parameter inputs to $\alpha_{T}$.

\begin{tabular}{ll}
\hline \hline Parameter & \multicolumn{1}{c}{$\Delta \alpha_{T}$} \\
\hline Meson production ratio, $r_{K / \pi}=0.149 \pm 0.06[21]$ & 0.007 \\
Mean effective temperature, & 0.0051 \\
$\quad\left\langle T_{\text {eff }}\right\rangle=221.93 \pm 0.32 \mathrm{~K}$ & \\
Threshold energy, $\left\langle E_{\text {th }} \cos \theta\right\rangle=0.795 \pm 0.14 \mathrm{TeV}$ & 0.0048 \\
Kaon constant, $B_{K}^{1}=1.740 \pm 0.028$ & 0.00046 \\
Pion constant, $B_{\pi}^{1}=1.460 \pm 0.007$ & 0.000063 \\
Total & 0.010 \\
\hline \hline
\end{tabular}

In all, $5.7 \%$ of the data set survived the cuts for both the forward and reverse field detector configurations. For the charge-separated samples linear regressions yielded effective temperature coefficients, $\alpha_{T}\left(\mu^{+}\right)=0.79 \pm 0.05$ and $\alpha_{T}\left(\mu^{-}\right)=0.77 \pm 0.06$ with $\chi^{2} / N_{\text {d.o.f. }}$ of $1933 / 1758$ and $1688 / 1751$ respectively. These numbers are consistent with each other, so there is no measurable difference between the temperature effect on $\mu^{+}$and $\mu^{-}$. The value of the charge-separated $\alpha_{T}$ is expected to be smaller than the previous $\alpha_{T}$ with no charge separation because the selection cuts change the energy distribution over which the integration is performed to calculate $\alpha_{T}$. This can be seen in Fig. 3, with the most dramatic difference between the all muon and charge-separated distributions of $E_{\mu} \cos \theta$. This difference could produce the systematic offset observed between these values, and is discussed further in the next section.

\section{DISCUSSION}

\section{A. Predicted $\boldsymbol{\alpha}_{T}$}

The theoretical prediction of $\alpha_{T}$ can be written as [2]

$$
\alpha_{T}=-\frac{E_{\mathrm{th}}}{I_{\mu}^{0}} \frac{\partial I_{\mu}}{\partial E_{\mathrm{th}}}-\gamma .
$$

Using the differential muon intensity [20],

$$
\begin{aligned}
\frac{d I_{\mu}}{d E_{\mu}}= & \int_{0}^{\infty} \mathcal{P}_{\mu}(E, X) d X \\
\simeq & A \times E^{-(\gamma+1)}\left(\frac{1}{1+1.1 E_{\mu} \cos \theta / \epsilon_{\pi}}\right. \\
& \left.+\frac{0.38 r_{K / \pi}}{1+1.1 E_{\mu} \cos \theta / \epsilon_{K}}\right),
\end{aligned}
$$

and the MACRO approximation for the muon intensity [3], the prediction for $\alpha_{T}$ can be calculated:

$$
\alpha_{T}=\frac{1}{D_{\pi}} \frac{1 / \epsilon_{K}+A_{K}^{1}\left(D_{\pi} / D_{K}\right)^{2} / \epsilon_{\pi}}{1 / \epsilon_{K}+A_{K}^{1}\left(D_{\pi} / D_{K}\right) / \epsilon_{\pi}}
$$

where

$$
D_{\pi, K}=\frac{\gamma}{\gamma+1} \frac{\epsilon_{\pi, K}}{1.1 E_{\mathrm{th}} \cos \theta}+1
$$

Note that this can be reduced to MACRO's previously published expression $\left(\alpha_{T}\right)_{\pi}$ [3], by setting $A_{K}^{1}=0$ (no kaon contribution). $A_{K}^{1}=0.38 \cdot r_{K / \pi}$ is the same as in Sec. II.

A numerical integration using a Monte Carlo method was performed to find the expected value of the seasonal effect coefficient, $\left\langle\alpha_{T}\right\rangle_{p}$, for the far detector. A set of muons was generated by drawing values of $E_{\mu}$ and $\cos \theta$ separately from the differential intensity of muons at the surface, calculated in [20]. A random azimuthal angle, $\phi$, was assigned to each event and $\operatorname{combined}$ with $\cos \theta$ and 
TABLE III. Systematic errors on the theoretical parameter inputs to $\alpha_{T}$.

\begin{tabular}{lc}
\hline \hline Parameter & $\Delta \alpha_{T}$ \\
\hline Meson production ratio, $K / \pi=0.149 \pm 0.06[21]$ & 0.020 \\
Rock map uncertainty $\pm 10 \%$ & 0.013 \\
Muon spectral index, $\gamma=1.7 \pm 0.1$ & 0.0031 \\
Kaon critical energy, $\epsilon_{K}=0.851 \pm 0.014 \mathrm{TeV}$ & 0.0014 \\
Pion critical energy, $\epsilon_{\pi}=0.114 \pm 0.003 \mathrm{TeV}$ & 0.0002 \\
Theoretical total & 0.024 \\
\hline \hline
\end{tabular}

the Soudan rock overburden map [5] to find the slant depth, $S(\cos \theta, \phi)$, of the event. This was converted into the corresponding threshold energy, $E_{\mathrm{th}}$, required for a muon on the surface to propagate to the far detector. Events satisfying $E_{\mu}>E_{\mathrm{th}}$ were retained, and the mean value of $\alpha_{T}$ was found for a sample of 10000 events, giving $\left\langle\alpha_{T}\right\rangle_{p}=0.864 \pm 0.024$ for MINOS. When this calculation was performed using the lower energy charge-separated energy spectrum, the result is an $\left\langle\alpha_{T}\right\rangle_{p}$ value that is lower by 0.015 . This is most clearly seen in Eq. (13), which is dominated by the leading $1 / D_{\pi}$ term. As $E_{\text {th }} \cos \theta$ increases, $D_{\pi}$ goes to one. Any selection that reduces the $E_{\text {th }} \cos \theta$ distribution will then reduce the expected $\alpha_{T}$.

The systematic uncertainty on $\left\langle\alpha_{T}\right\rangle_{p}$ was found by modifying the input parameters and recalculating $\alpha_{T}$. The dominant contributions were from: (a) the \pm 0.06 uncertainty in meson production ratio; (b) the $\pm 10 \%$ in rock map uncertainty ${ }^{1}$; (c) the \pm 0.1 uncertainty in muon spectral index; (d) the $\pm 0.014 \mathrm{TeV}$ uncertainty in kaon critical energy; and (e) the $\pm 0.003 \mathrm{TeV}$ uncertainty in pion critical energy. These uncertainties are summarized in Table III.

Figure 7 shows effective temperature coefficients from MINOS and other underground experiments, including those of the MACRO survey [3], as a function of detector depth. The MINOS and Sherman [15] effective temperature coefficients shown in Fig. 7 were calculated using Eq. (10). The other experimental data points are taken from the MACRO survey [3] and were calculated using a definition which excluded the contributions from kaons and were limited by temperature measurements up to $20 \mathrm{~g} / \mathrm{cm}^{2}$; when the MINOS result is recalculated with this definition the effective temperature coefficient de-

\footnotetext{
${ }^{1}$ The rock map is not a determination of the slant depth by geophysical means. It was created by measuring the muon flux coming from a particular solid angle region on the sky and then normalizing to the all-world Crouch underground muon intensity curve [25]. This was done with both Soudan 2 data [26] and with MINOS data [5], and these calculations were shown to agree to within $10 \%$. Average cosmic ray muon flux, like those determined here and in [5] can be determined using this method, although in any particular direction the rock map can be much different from what was calculated (e.g., in the direction of iron veins).
}

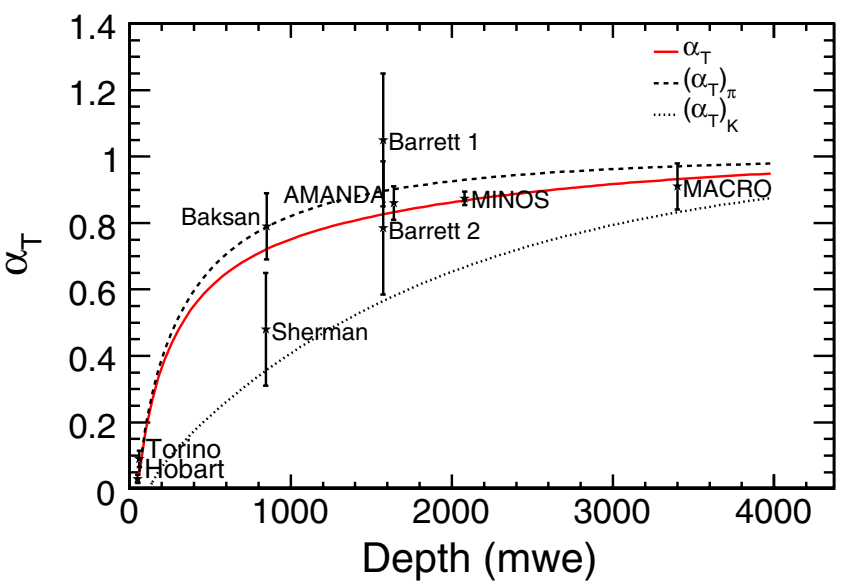

FIG. 7 (color online). The theoretical prediction for $\alpha_{T}$ as a function of detector depth. The dashed (top) curve is the prediction using the pion-only model (of MACRO) and the dotted (bottom) curve is the prediction using a kaon-only model. The solid (middle) curve is the new prediction including both $K$ and $\pi$. These curves are illustrative only as the definition of effective temperature used to calculate the experimental values also depends on the $K / \pi$ ratio. The data from other experiments are shown for comparison only, and are from Barrett 1, 2 [2], AMANDA [4], MACRO [11], Torino [12], Sherman [15], Hobart [16] and Baksan [17].

creases to $\alpha_{T}=0.835$. To compare the experimental values with the theoretical model, Eq. (13), the expected effective temperature coefficient as a function of depth was calculated using the numerical integration method outlined earlier, using standard rock and a flat overburden, and is shown in Fig. 7 as the solid line. There is qualitative agreement between the prediction and the experimentally measured values, but quantitative comparisons would require recalculating the experimental values using the kaoninclusive definition of effective temperature. The two dashed lines in Fig. 7 show the effective temperature dependence for the extreme pion-only and kaon-only predictions. Figure 7 is illustrative only, as the dependence of the experimentally measured effective temperature coefficient on the input $K / \pi$ ratio is not explicitly shown.

\section{B. Measurement of atmospheric $K / \pi$ ratio}

The uncertainty on the atmospheric $K / \pi$ ratio in the current cosmic ray flux models is of order $40 \%$ [21]. There has not been a measurement of this ratio with cosmic rays. Previous measurements have been made at accelerators for $p+p$ collisions [27], $\mathrm{Au}+\mathrm{Au}$ collisions [28], $\mathrm{Pb}+\mathrm{Pb}$ collisions [29,30] and $p+\bar{p}$ collisions [31]. Many other older measurements are summarized in [32]. The experimental and theoretical values of $\alpha_{T}$ can be combined to give a new measurement of the $K / \pi$ ratio for the reaction $p+A_{\text {atm }}$, with $E_{p} \gtrsim 7 \mathrm{TeV}$. The threshold muon surface energy, $E_{\mathrm{th}}=0.73 \mathrm{TeV}$ and the median muon surface energy, $\left\langle E_{\mu}\right\rangle$, is $1.15 \mathrm{TeV}$. On average, the muon energy 


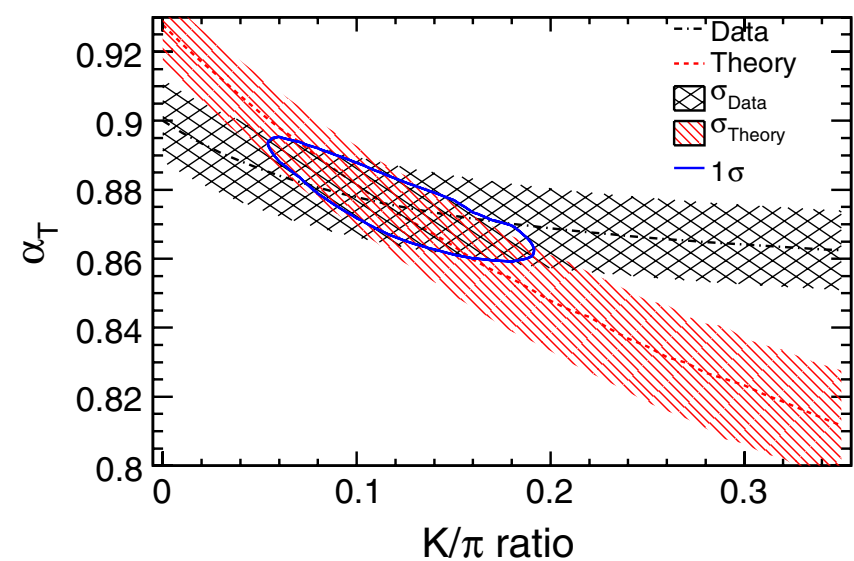

FIG. 8 (color online). The MINOS experimental $\alpha_{T}$ as a function of the $K / \pi$ ratio (dot-dashed line), with its error given by the crosshatched region, on the same axes as the theoretical $\alpha_{T}$ as a function of the $K / \pi$ ratio (dashed line), with its error given by the hatched region. The error on the experimental $\alpha_{T}$ (from Table II $E_{\text {th }} \cos \theta, B_{\pi, K}^{1}$ and $\left\langle T_{\text {eff }}\right\rangle$ ) plus statistical error is \pm 0.012 , and the theoretical $\alpha_{T}$ error (from $\epsilon_{\pi, K}$ and the rock map, Table III) is \pm 0.013 at the best fit point. The intersection is at $K / \pi=0.12_{-0.05}^{+0.07}$. The solid line denotes the $1 \sigma$ contour around the best fit.

is one-tenth the energy of its parent primary. The theoretical $\alpha_{T}$ depends directly on the $K / \pi$ ratio, as a consequence of the different interaction and decay properties of kaons and pions in the atmosphere. Since kaons and pions have different critical energies and attenuation lengths, the effective temperature also depends on the $K / \pi$ ratio, and therefore the experimental $\alpha_{T}$ is a weak function of the $K / \pi$ ratio. By plotting the experimental and theoretical values of $\alpha_{T}$ as functions of the $K / \pi$ ratio and finding the intersection of the two curves, a measurement of the $K / \pi$ ratio can be obtained.

Figure 8 shows the experimental and theoretical values of $\alpha_{T}$ as a function of the $K / \pi$ ratio for the MINOS data. The errors in the experimental and theoretical values of $\alpha_{T}$ are taken to be \pm 0.012 and \pm 0.013 respectively, obtained by combining the statistical errors in quadrature with the systematic errors in Tables II and III, but omitting the error in the $K / \pi$ ratio in each case. The error on the theoretical value of $\alpha_{T}$ grows with increasing $K / \pi$ ratio because $\epsilon_{K}$ has a larger uncertainty than $\epsilon_{\pi}$, so a larger contribution from kaons introduces more uncertainty. The intersection of the two curves occurs at $K / \pi=0.12_{-0.05}^{+0.07}$. The uncertainty is estimated by assuming Gaussian errors for the theoretical and experimental values of $\alpha_{T}$ and performing a $\chi^{2}$ minimization to determine the $\Delta \chi^{2}=1$ contour that encompasses the best fit point.

Previous measurements of the $K / \pi$ ratio do not directly compare to this indirect measurement. Nevertheless, the central value of MINOS' measurement is consistent with the collider-based direct measurements, although the indirect error bars are larger than those on the direct measure-

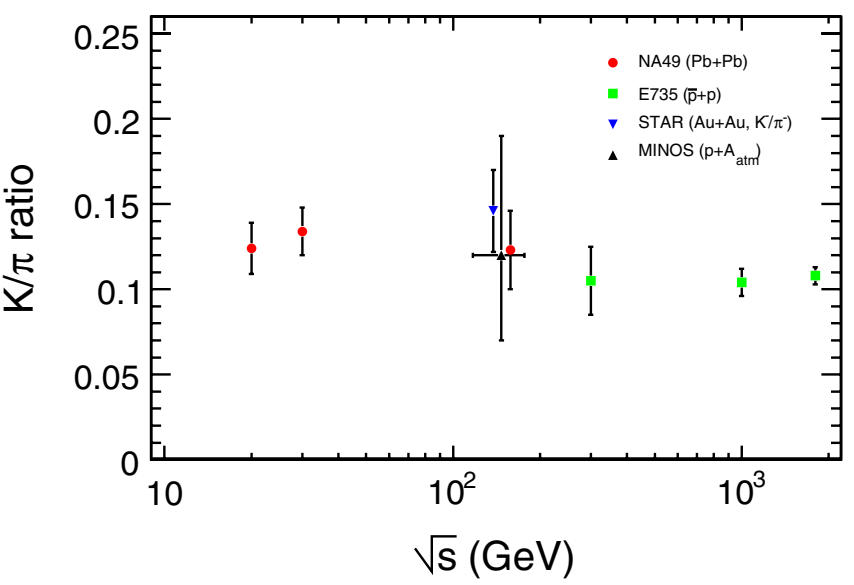

FIG. 9 (color online). A compilation of selected measurements of $K / \pi$ for various center of mass energies. The STAR value was from $\mathrm{Au}+\mathrm{Au}$ collisions at RHIC [28], the NA49 measurement was from $\mathrm{Pb}+\mathrm{Pb}$ collisions at SPS [29,30], and the E735 measurement was from $p+\bar{p}$ collisions at the Tevatron [31].

ments. A comparison of this measurement to other measurements is shown in Fig. 9. Only the MINOS result is for a reaction where the interacting particles do not have equivalent energy in the laboratory frame. Nevertheless, they are all presented on the same axes for a broad overview. The central value of MINOS' indirect cosmic-raybased $K / \pi$ measurement is consistent with the colliderbased direct measurements, and the associated error bars span the dispersion in those direct measurements.

\section{CONCLUSIONS}

A 5 yr sample of $67.32 \times 10^{6}$ cosmic ray induced muons has been collected by the MINOS far detector and daily rate fluctuations have been compared to daily fluctuations in atmospheric temperature. These distributions were shown to be highly correlated, with a correlation coefficient of 0.90 . The constant of proportionality relating the two distributions, $\alpha_{T}$, was found to be $0.873 \pm$ 0.009 (stat) \pm 0.010 (syst). This value is in good agreement with the theoretical expectation of $\left\langle\alpha_{T}\right\rangle=0.864 \pm 0.024$. A measurement of the temperature dependence of the rate of $\mu^{+}$separate from $\mu^{-}$was performed for the first time. There is no statistically significant difference between $\alpha_{T}\left(\mu^{+}\right)$and $\alpha_{T}\left(\mu^{-}\right)$.

The experimental value of $\alpha_{T}$ for the combined muon sample has the lowest uncertainty of any such measurement. While other experiments have estimated the effect of atmospheric temperature on kaon induced muons [2,3], this is the first result to quantify the kaon-inclusive effective temperature coefficient. The new kaon-inclusive model fits the MINOS far detector data better than the pion-only model [3] and suggests a measurement of the atmospheric $K / \pi$ ratio. Applying the differing temperature variations of kaon and pion decay to the seasonal variations 
analysis allowed the first measurement of the atmospheric $K / \pi$ ratio for $E_{p} \geq 7 \mathrm{TeV}$. It was found to be $K / \pi=$ $0.12_{-0.05}^{+0.07}$

\section{ACKNOWLEDGMENTS}

We thank the Fermilab staff and the technical staffs of the participating institutions for their vital contributions. This work was supported by the U.S. Department of Energy, the U.K. Science and Technologies Facilities Council, the U.S. National Science Foundation, the State and University of Minnesota, the Office of Special Accounts for Research Grants of the University of
Athens, Greece, FAPESP (Fundacao de Amparo a Pesquisa do Estado de Sao Paulo) and CNPq (Conselho Nacional de Desenvolvimento Cientifico e Tecnologico) in Brazil. We gratefully acknowledge the Minnesota Department of Natural Resources for their assistance and for allowing us access to the facilities of the Soudan Underground Mine State Park and the crew of the Soudan Underground Physics Laboratory for their tireless work in building and operating the MINOS far detector. We also acknowledge the BADC and the ECMWF for providing the environmental data for this project.
[1] S. Osprey et al., Geophys. Res. Lett. 36, L05809 (2009).

[2] P. Barrett et al., Rev. Mod. Phys. 24, 133 (1952).

[3] M. Ambrosio et al. (MACRO Collaboration), Astropart. Phys. 7, 109 (1997).

[4] A. Bouchta (AMANDA Collaboration), in Proceedings of the 26th International Cosmic Ray Conference, Salt Lake City, 1999, Vol. 2 (AIP, Salt Lake City, UT, 2000), p. 108.

[5] P. Adamson et al. (MINOS Collaboration), Phys. Rev. D 76, 052003 (2007).

[6] P. Adamson et al. (MINOS Collaboration), Phys. Rev. D 77, 072002 (2008).

[7] P. Adamson et al. (MINOS Collaboration), Phys. Rev. D 73, 072002 (2006).

[8] D. G. Michael et al. (MINOS Collaboration), Nucl. Instrum. Methods 596, 190 (2008).

[9] B. J. Rebel, Ph.D. Dissertation, Indiana University [Institution Report No. FERMILAB-THESIS-2004-33, 2004].

[10] European Centre for Medium-Range Weather Forecasts ECMWF Operational Analysis data, [Internet] British Atmospheric Data Centre 2006-2007 (available from http://badc.nerc.ac.uk/data/ecmwf-op/).

[11] M. Ambrosio et al. (MACRO Collaboration), Phys. Rev. D 67, 042002 (2003).

[12] G. Cini Castagnoli and M. Dodero, Nuovo Cimento B 51, 525 (1967).

[13] J. Humble et al., in Proceedings of the 16th International Cosmic Ray Conference, Kyoto, 1979 (Institute of Cosmic Ray Research, Tokyo, 1979), Vol. 4, p. 258.

[14] D. Cutler et al., in Proceedings of the 17th International Cosmic Ray Conference, Paris, 1981 (Centre d'Etudes Nucleaires, Saclay, 1981), Vol. 4, p. 290.

[15] N. Sherman, Phys. Rev. 93, 208 (1954).
[16] A. G. Fenton, R. M. Jacklyn, and R. B. Taylor, Nuovo Cimento B 22, 285 (1961).

[17] Y. Andreyev et al., in Proceedings of the 20th International Cosmic Ray Conference, Moscow, 1987 (Nauka, Moscow, 1987), Vol. 3, p. 270.

[18] E.W. Grashorn, Ph.D. Dissertation, University of Minnesota [Institution Report No. FERMILAB-THESIS2008-06, 2008].

[19] E. W. Grashorn et al., arXiv:0909.5382.

[20] T.K. Gaisser, Cosmic Rays and Particle Physics (Cambridge University Press, Cambridge, England, 1990).

[21] G. D. Barr, T. K. Gaisser, S. Robbins, and T. Stanev, Phys. Rev. D 74, 094009 (2006).

[22] S. P. Ahlen et al. (MACRO Collaboration), Nucl. Phys. B370, 432 (1992).

[23] I. Durre, R. S. Vose, and D. Wuertz, J. Clim. 19, 53 (2006).

[24] F. James and M. Roos, Comput. Phys. Commun. 10, 343 (1975).

[25] M. Crouch, in Proceedings of the 20th International Cosmic Ray Conference, Moscow, 1987 (Ref. [17]), Vol. 6, p. 165.

[26] S. M. Kasahara, Ph.D. thesis, University of Minnesota [Institution Report No. UMI-97-21625, 1997].

[27] A. M. Rossi et al., Nucl. Phys. B84, 269 (1975).

[28] C. Adler et al. (STAR Collaboration), Phys. Lett. B 595, 143 (2004).

[29] S. V. Afanasiev et al. (NA49 Collaboration), Phys. Rev. C 66, 054902 (2002).

[30] C. Alt et al. (NA49 Collaboration), Eur. Phys. J. C 45, 343 (2006).

[31] T. Alexopoulos et al. (E735 Collaboration), Phys. Rev. D 48, 984 (1993).

[32] M. Gazdzicki and D. Roehrich, Z. Phys. C 65, 215 (1995). 